\title{
The relationship between geophysical conditions and ELF amplitude in modulated heating experiments at HAARP: Modeling and experimental results
}

\author{
G. Jin, ${ }^{1}$ M. Spasojevic, ${ }^{1}$ M. B. Cohen, ${ }^{1}$ U. S. Inan, ${ }^{1,2}$ and N. G. Lehtinen ${ }^{1}$ \\ Received 16 March 2011; revised 25 April 2011; accepted 2 May 2011; published 26 July 2011.
}

[1] Experiments for generating extremely low frequency (ELF) radio waves using modulated HF heating of the auroral ionosphere have been conducted and refined at the High Frequency Active Auroral Research Program (HAARP) facility at Gakona, Alaska. Because this technique is dependent on strength of the naturally generated electrojet current system, the amplitude of the generated ELF changes with geophysical conditions. Past work has shown that electrojet current strength as measured by magnetometers often correlates with generated ELF amplitude, but there are periods of poor or negative correlation. We attempt to use additional diagnostics from a radar, riometer, ionosonde, and magnetometer chain to understand how ionospheric conditions affect ELF generation. We then present the results of a statistical model that shows that ELF amplitude is roughly proportional to magnetometer measurements for a fixed value of riometer absorption and that the proportionality constant decreases as riometer absorption increases. Theoretical simulations of modulated heating are conducted for a variety of ionospheric density profiles to verify that denser profiles result in smaller gains for ELF generation as a function of electrojet current at a given electric field.

Citation: Jin, G., M. Spasojevic, M. B. Cohen, U. S. Inan, and N. G. Lehtinen (2011), The relationship between geophysical conditions and ELF amplitude in modulated heating experiments at HAARP: Modeling and experimental results, J. Geophys. Res., 116, A07310, doi:10.1029/2011JA016664.

\section{Introduction}

[2] Experiments involving generation of extremely low frequency (ELF, up to $3 \mathrm{kHz}$ ) and very low frequency (VLF, 3-30 kHz) electromagnetic waves using modulated ionospheric heating have been conducted since Getmantsev et al. [1974]. The technique of using high-frequency (HF, 3-30 MHz) radiation to modulate the temperature and conductivity of the $D$ region ionosphere has the benefit of utilizing the natural current systems existing in the upper atmosphere, thus easing the difficulties of creating a current source large enough to radiate at such long wavelengths. The High Frequency Active Auroral Research Program (HAARP) heating facility in Gakona, Alaska (geographic: $62.39^{\circ} \mathrm{N}, 145.15^{\circ} \mathrm{W}$, geomagnetic: $63.0^{\circ} \mathrm{N}, \mathrm{MLT}=$ $\mathrm{UT}+10.8$ ) is currently the most powerful such heater.

[3] Modulated $D$ region heating still suffers from low efficiency despite the high power available at HAARP (3.6 MW > 1 GW ERP) and new techniques have been discovered for increasing the power of the generated ELF waves. For example, a new geometric modulation technique

\footnotetext{
${ }^{1}$ Department of Electrical Engineering, Stanford University, Stanford, California, USA.

${ }^{2}$ Department of Electrical Engineering, Koç University, Istanbul, Turkey.

Copyright 2011 by the American Geophysical Union. 0148-0227/11/2011JA016664
}

described by Cohen et al. [2010b] has shown improvements of $\sim 10 \mathrm{~dB}$ in received amplitude when compared to simple amplitude modulation. However, natural changes in the electrojet are often the dominant source of variability in ELF generation and concerns over the reliability of modulated heating as an ELF source center around this variability. Data presented by Rietveld et al. [1987] and Oikarinen et al. [1997] show natural amplitude fluctuations of at least $12 \mathrm{~dB}$. It is usually assumed that natural changes in ELF amplitude correlate directly with changes in electrojet current strength. Magnetometers provide a simple way for measuring the electrojet strength by reporting 3 components of the Earth's magnetic field: typically $H$ in the magnetic northward direction, $D$, in the magnetic eastward direction, and $Z$ in the vertical direction. Kamide et al. [1982] showed the total overhead east-west current is linearly proportional to $\Delta H$, the difference between the measured value of $H$ and its undisturbed value during quiet conditions. However, experimental data often shows periods where electrojet current strength and ELF amplitude are poorly or negatively correlated [Rietveld et al., 1987; Oikarinen et al., 1997; Jin et al., 2009].

[4] The earliest attempts to correlate ELF generation with other measurements were shown by Kapustin et al. [1977], who noted a positive, linear correlation between ELF amplitude and magnetometer measurements but did not quantify the relationship. Rietveld et al. [1983, 1987], and Oikarinen et al. [1997] present many more examples from 
the ionospheric heater at Tromsø noting cases of positive, weak, and negative correlation between ELF intensity and magnetometer data. Rietveld et al. [1987] attempt to correlate ELF intensity with riometer absorption and the electric field driving the electrojet as measured by STARE. Correlation between ELF amplitude and STARE was always positive with correlation coefficients ranging from 0.17 to 0.89 . Correlation coefficients for $\Delta H$ were mixed ranging from -0.23 to 0.93 , and correlation with riometer absorption was worse. However, the riometer-ELF correlation coefficients with the highest magnitude were always negative. Rietveld et al. [1987] mention that this negative correlation may be the result of lower electric fields that accompany periods of high absorption. Jin et al. [2009] showed statistical data where ELF amplitude is positively and linearly correlated with $\Delta H$ in $55 \%$ of cases. However, this proportionality constant changes depending on whether conditions were disturbed. The proportionality constant is larger in time intervals when $\Delta H$ is small, and smaller when $\Delta H$ is larger.

[5] Modeling can provide insight into why the relationship between the electrojet and ELF strength changes. Barr and Stubbe [1984] found that different ionospheric electron density profiles had very subtle effects on both generation of the current moment and propagation in the Earth-ionosphere waveguide. While higher densities resulted in less excitation of the ionosphere, this effect was compensated for by decreasing source altitudes and better waveguide excitation suggesting little overall effect of electron density on measured ELF amplitude.

[6] In this paper, we assemble a large collection of ELF measurements taken during HAARP experiments and examine how they relate to other ionospheric diagnostics such as magnetometer, riometer, radar and ionosonde data. We construct a statistical neural network model using the riometer, magnetometer, and ionosonde data to predict ELF intensity. Finally we use a HF heating model and full-wave propagation model to explore how received ELF fields and magnetometer measurements would be affected by different electron density profiles and electric fields and compare the results to the statistical model.

\section{Data Set and Observations}

[7] To measure the strength of the generated ELF signals, ELF data is taken from an AWESOME ELF/VLF receiver [Cohen et al., 2010c] located in Chistochina, Alaska $37 \mathrm{~km}$ northeast of HAARP. The receiver processes signals from two orthogonal loop antennas and can detect signal levels as low as 1 fT. We use data taken from 2007 and 2008 when HAARP was transmitting with a full-power $(3600 \mathrm{~kW})$, vertical, X-mode beam, modulated at $2125-2130 \mathrm{~Hz}$ with a $40-50 \%$ duty cycle. This totals $2596 \mathrm{~min}$ of experiment time over 20 days during nighttime at $100 \mathrm{~km}$ altitude, and 2127 min over 20 days during daytime. Because HAARP experiment formats typically use pulses of a few seconds at each frequency, ELF amplitude is taken by mixing each pulse at 2125 or $2130 \mathrm{~Hz}$ to baseband then taking the median amplitude. The amplitudes from each antenna are combined to yield the magnitude of the horizontal component of the wave magnetic field in pT. The time resolution is then the period of the format, typically $30 \mathrm{~s}$.
There are 6262 such pulses during nighttime and 4328 during daytime.

[8] To estimate the strength of the east-west electrojet current, we use data from the Geophysical Institute Magnetometer Array [Wilkinson and Heavner, 2006]. The Gakona magnetometer is located at the HAARP facility. It samples the $H, D$, and $Z$ components of the magnetic field once per second. An estimate of the quiet time magnetic field is made from the average value of $H$ on nearby quiet days and subtracted from the magnetometer's $H$ measurement to yield $\Delta H$. The value of $\Delta H$ at HAARP is used as an estimate of the overhead current, i.e., the current available for HAARP to modulate. However, at times strong currents at further distances (primarily to the north as HAARP is typically located equatorward of peak electrojet) will produce magnetic perturbations on the Gakona magnetometer, but they would not be subject to modulation using HAARP. To explore how the spatial distribution of the currents affects ELF generation, we use the Geophysical Institute's Geospace Environment Data Display System (GEDDS) which provides estimates of equivalent horizontal currents at latitudes in one degree increments from $59^{\circ} \mathrm{N}$ to $71^{\circ} \mathrm{N}$ [Sun et al., 1993] with $1 \mathrm{~min}$ time resolution based on measurements from a chain of 6 magnetometers.

[9] Riometer data is used to infer changes in the $D$ and $E$ region ionospheric densities and is taken from the HAARP classic riometer, which receives radio noise from the sky at $30 \mathrm{MHz}$. The quiet day curve containing the expected signal from the detector in the absence of ionospheric absorption is subtracted from the actual detector signal to yield the absorption in $\mathrm{dB}$. Interference can result in negative absorption values when the detector signal is greater than the quiet day curve. These values are clipped to $0 \mathrm{~dB}$. Data are sampled every minute.

[10] Ionosonde data is also used to estimate changes in the $E$ region density. The HAARP ionosonde provided by the University of Massachusetts at Lowell uses automated algorithms to determine ionospheric parameters from ionograms. Of interest here are the $E$ and sporadic $E$ critical frequencies $\left(f_{0} E\right.$ and $\left.f_{0} E s\right)$. Although such automated processing is not completely reliable, assembling a large number of observations may reveal trends in how $E$ region parameters affect ELF generation. The time resolution of the ionosonde data set is $15 \mathrm{~min}$ so the relevant value corresponding to each ELF pulse is defined as the value of the closest 15 min interval if it is given. Using this technique, $46 \%$ of the ELF receptions in this data set are associated with sporadic $E$ values. The software did not report $f_{0} E$ for any of the nighttime cases.

[11] An estimate of the electric field above HAARP would be an ideal diagnostic for examining ELF generation efficiency. As part of the Super Dual Auroral Radar Network (SuperDARN), the coherent scatter HF radar at Kodiak provides coverage over HAARP. These radars require the presence of ionospheric density irregularities to act as scatterers in order to determine the electric field, but unfortunately irregularities are absent at Kodiak for almost all of the ELF experiments conducted at HAARP. Thus, in order to estimate the local electric field, we use global convection maps derived from the SuperDARN array [Ruohoniemi and Baker, 1998; Shepherd and Ruohoniemi, 2000]. In this approach, ionospheric convection velocities derived from the SuperDARN network are fitted to an 

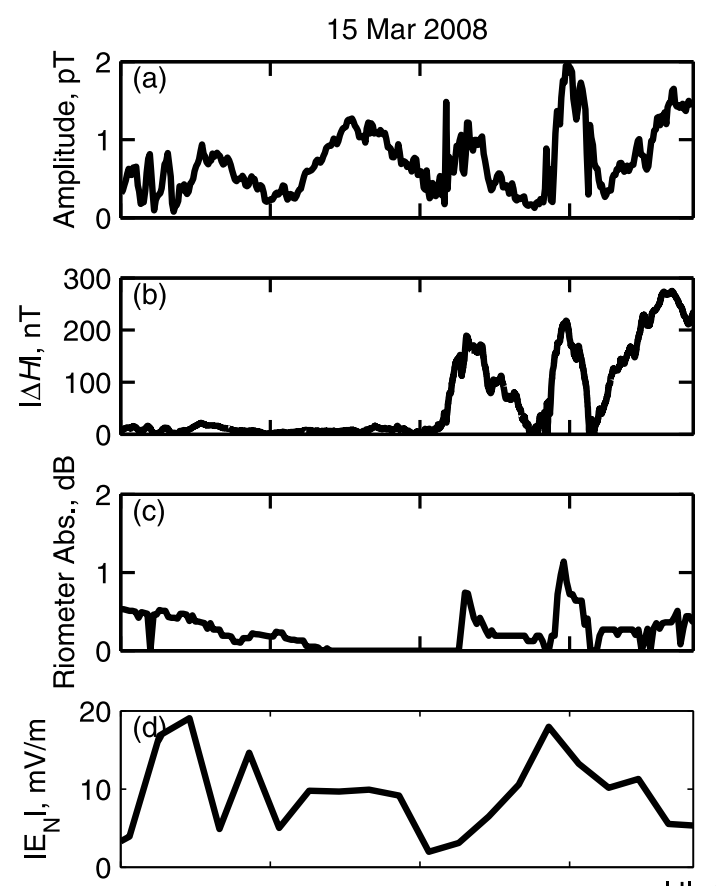

(e) $|\mathrm{J}|, \mathrm{A} / \mathrm{m}$

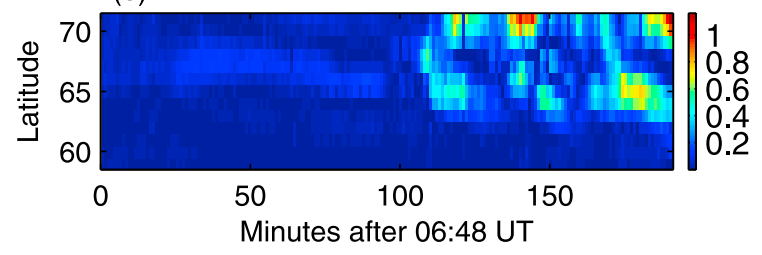

Figure 1. Plots of (a) $2125 \mathrm{~Hz}$ ELF amplitude at Chistochina, Alaska, from 06:48 to 09:59 UT and (b) $|\Delta H|$ at Gakona, Alaska, over the same period showing an increase in activity at 08:36 UT. The increase in ELF amplitude after 08:36 UT is less dramatic. (c) Riometer data show low absorption immediately before 08:36 UT and high absorption afterward. (d) Northward component of the electric field from SuperDARN is not substantially stronger after 08:36 UT than before. (e) Electrojet intensifies substantially after 08:36 but mostly at higher latitudes.

expansion of the electrostatic potential in terms of spherical harmonic functions. A statistical model, which is dependent on the IMF direction, is used to constrain the solution in regions where no SuperDARN data are available. The resulting convection maps are optimized toward large-scale convection patterns and are not ideal for resolving small-scale features over HAARP [Chisham et al., 2007]. The local electric field at HAARP is interpolated from the global convection map and the northward component of the field is available every $10 \mathrm{~min}$.

[12] Figure 1 summarizes data from each of the instruments. The data are from an approximately $3 \mathrm{~h}$ experiment starting on 15 March 2008 at 06:48 UT. The panels from top to bottom show (Figure 1a) ELF amplitude at Chistochina, (Figure 1b) $\Delta H$ from the Gakona magnetometer, (Figure 1c) riometer absorption at HAARP, (Figure 1d) the magnitude of the estimated northward component of the electric field at HAARP from the SuperDARN network $\left(\left|E_{N}\right|\right)$, (Figure 1e) the spatial distribution of the electrojet current density $(J)$ derived from the magnetometer array. There were only a few points where the ionosonde detected a sporadic $E$ layer so $f_{0} E s$ is not shown. Near 08:36 UT a substorm occurred with $\Delta H$ approaching $300 \mathrm{nT}$. However, ELF amplitude during the substorm only reaches $2 \mathrm{pT}$ compared to amplitudes around $1 \mathrm{pT}$ during the quiet period. Riometer absorption is very low in the hour before substorm onset, then increases to $\sim 1 \mathrm{~dB}$. Electric field is varying throughout. The electrojet currents in Figure 1e also intensify during the substorm but mostly at higher latitudes between $65^{\circ} \mathrm{N}$ to $70^{\circ} \mathrm{N}$. ELF generation is correlated with $|\Delta H|$ both before (correlation coefficient $=0.33$ ) and after (correlation coefficient $=0.74)$ the substorm. While the correlation coefficient of 0.33 before the substorm shows a weak correlation, the probability of error is much less than 0.05 indicating that the correlation is still statistically significant. The proportionality constant $(0.18 \mathrm{pT} / \mathrm{nT}$ before the substorm and $0.01 \mathrm{pT} / \mathrm{nT}$ after) is clearly different. This disparity and its relationship to the larger values of riometer absorption and other ionospheric parameters during the substorm will be quantified with statistical and theoretical models.

\section{Statistical Model}

\subsection{Methodology}

[13] In order to further examine the efficiency of ELF generation, we create a statistical model of ELF strength as a function of multiple ionospheric parameters. We use a neural network to fit the detected ELF signal strength at Chistochina to different combinations of magnetometer, riometer, $E$ and sporadic $E$ critical frequency, and electric field inputs. A neural network is one technique for fitting a multidimensional input data set to an output set. Once the network is trained by fitting known measurements, it can then estimate the ELF strength when given new values for the inputs.

[14] The neural network used here consists of a layer of 10 hidden neurons and an output layer of a single neuron. The hidden layer neurons each receive the inputs, find a weighted sum, apply a bias, and pass them through a hyperbolic tangent function. The output neuron receives the outputs from the hidden layer neurons, weighs and biases them, and then passes the result through a linear function whose output is the output of the model. The weights and biases are determined with a training algorithm on data randomly selected from $70 \%$ of the input data with the remaining $30 \%$ used for testing and validation. Training involves choosing a set of weights and biases that minimizes the network's squared error (the squared difference between the network's output and the measured data). This can be a difficult problem because of the large number of parameters that must be adjusted. For example, the 10 neurons in the hidden layer with 2 inputs have 20 weights and 10 bias values that must be chosen. Several algorithms can be used to find a local minimum for the network's error over this 30 dimensional space. These are usually iterative algorithms that start at some value for the network's parameters, choose a new value for the parameters (based on the gradient of the network's error function for example), and repeat until it meets some stopping criteria. We use the Levenberg-Marquadt training algorithm as it provides good performance and is fast for 
Table 1. Neural Networks Constructed, the Correlation Coefficient (r) Between Model Output and Measurements, and Number of ELF Receptions Available for Training

\begin{tabular}{|c|c|c|c|c|}
\hline \multirow[b]{2}{*}{ Inputs } & \multicolumn{2}{|r|}{ Night } & \multicolumn{2}{|r|}{ Day } \\
\hline & $\mathrm{r}$ & Number of Points & $\mathrm{r}$ & Number of Points \\
\hline$|\Delta H|,\left|E_{N}\right|$ & 0.81 & 6262 & 0.6 & 4322 \\
\hline $\mathrm{I} \Delta H \mathrm{I}$, Riometer & 0.87 & 6262 & 0.6 & 4326 \\
\hline$|\Delta H|$, Riometer, $\left|E_{N}\right|$ & 0.88 & 6262 & 0.67 & 4322 \\
\hline$|\Delta H|, f_{0} E$ & - & 0 & 0.79 & 1175 \\
\hline$|\Delta H|, f_{0} E s$ & 0.90 & 2875 & 0.85 & 922 \\
\hline$|\Delta H|, f_{0} E s$, Riometer & 0.94 & 2875 & 0.92 & 922 \\
\hline J, Riometer & 0.75 & 5209 & 0.73 & 3661 \\
\hline $\mathrm{J}, f_{0} E s$ & 0.88 & 2604 & 0.78 & 893 \\
\hline
\end{tabular}

the size of the network we use [Hagan and Menhaj, 1994]. See Fine [1999] for a good introduction to this neural network architecture including a summary of training algorithms. The Neural Network Toolbox in the MATLAB software package was used to perform the fitting.

[15] Neural networks have a disadvantage in that the weights and biases that compose the inner workings of the neural network provide no insight as to how the inputs affect the outputs. For example, multiple linear regression, an alternate technique for multidimensional fitting, assigns coefficients to each input and the magnitude and sign of these coefficients can indicate the significance of each input to the output and whether the input is directly or inversely related to the output. For neural networks, an alternative is to sweep one or two inputs at a time while holding the remaining ones constant. Plotting how the output changes with input can then reveal any relationships between the inputs and output. We choose to use neural networks because they have the advantage of scaling easily as the number of input vectors increase and fitting complex relationships between outputs and inputs where it is more difficult to achieve good results using other techniques such as multiple linear regression.

[16] We construct several neural networks each with ELF amplitude measured at Chistochina as the output and combinations of $|\Delta H|$ from the HAARP magnetometer, electrojet current from a magnetometer chain, $E$ and sporadic $E$ layer critical frequencies, and $\left|E_{N}\right|$ from SuperDARN as inputs. The results are summarized in Table 1 which lists the inputs to each network, the correlation coefficient between the model output and measured output of ELF amplitude and the number of data points available for training and testing the model.

[17] The statistical model using only $|\Delta H|$ and riometer absorption performs well with a correlation coefficient of 0.87 between the model output and measured ELF amplitudes for all nighttime data. The model using $|\Delta H|$ and $\left|E_{N}\right|$ also performs well with a correlation coefficient of 0.81 . Including all 3 instruments only increases the correlation to 0.88 indicating that a model using only a magnetometer and either the riometer or the electric field is sufficient. We choose to drop the electric fields and focus on models utilizing the magnetometer and riometer. Although a statistical model utilizing only the magnetometer and electric field was able to reproduce measured data reasonably well, the actual output of the model is difficult to interpret and not physically intuitive. We could not discern any clear relationship between the electric field and ELF generation, which may indicate that the SuperDARN data are not reliable for this purpose due to the lack of irregularities above HAARP. A time resolution of $10 \mathrm{~min}$ also forced us to interpolate which may increase errors further though both linear and cubic spline interpolation were tried.

[18] A similar model using sporadic $E$ critical frequency measurements from the HAARP ionosonde instead of riometer absorption performed slightly better in nighttime cases when this measurement was available, resulting in a correlation coefficient of 0.90 . When combined with riometer data, a correlation coefficient of 0.94 was achieved.

[19] The daytime model using $|\Delta H|$ and riometer has worse performance than the nighttime model with a correlation coefficient near 0.6. Including $E$ and sporadic $E$ critical frequency in the model improves the correlation but only over a very narrow range of input values. The largest daytime value for $|\Delta H|$ was only $61 \mathrm{nT}$ when only including cases with sporadic $E$ data and only $50 \mathrm{nT}$ when only including cases with $E$ layer data.

[20] A concern with relying on the HAARP magnetometer is detection of strong currents much further north than HAARP that would not contribute to ELF generation. Figure 2a shows the intensity of the current at each of these latitudes for HAARP transmissions which resulted in ELF amplitudes along the $\mathrm{x}$ axis. The ELF amplitudes are sorted by amplitude. When ELF amplitudes were stronger (toward the right), the currents not only intensify but also move southward. To ensure that the magnetometer is a reasonable measure of the overhead current we plot the current at HAARP's latitude versus $|\Delta H|$ measured with the Gakona magnetometer in Figure 2b. ELF amplitude is shown in color. The correlation between the two is strong, indicating that the magnetometer is an acceptable diagnostic of the overhead currents although there are still points where the magnetometer overestimates the overhead current. How-

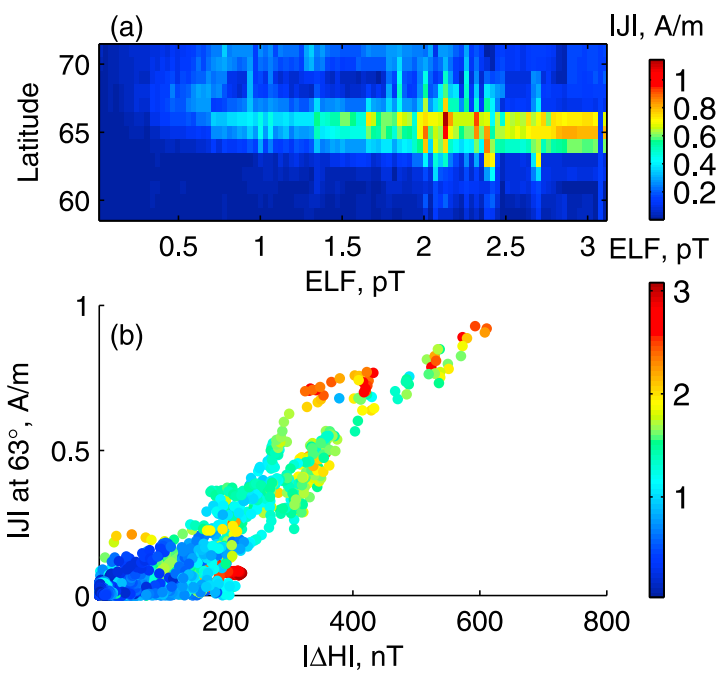

Figure 2. (a) Median electrojet current over latitude in cases where ELF intensity was in a given bin. (b) Electrojet current at HAARP's latitude plotted against $|\Delta H|$ showing generally good correlation, but with cases where $|\Delta H|$ is high compared to the overhead current. ELF intensity is shown in color. 

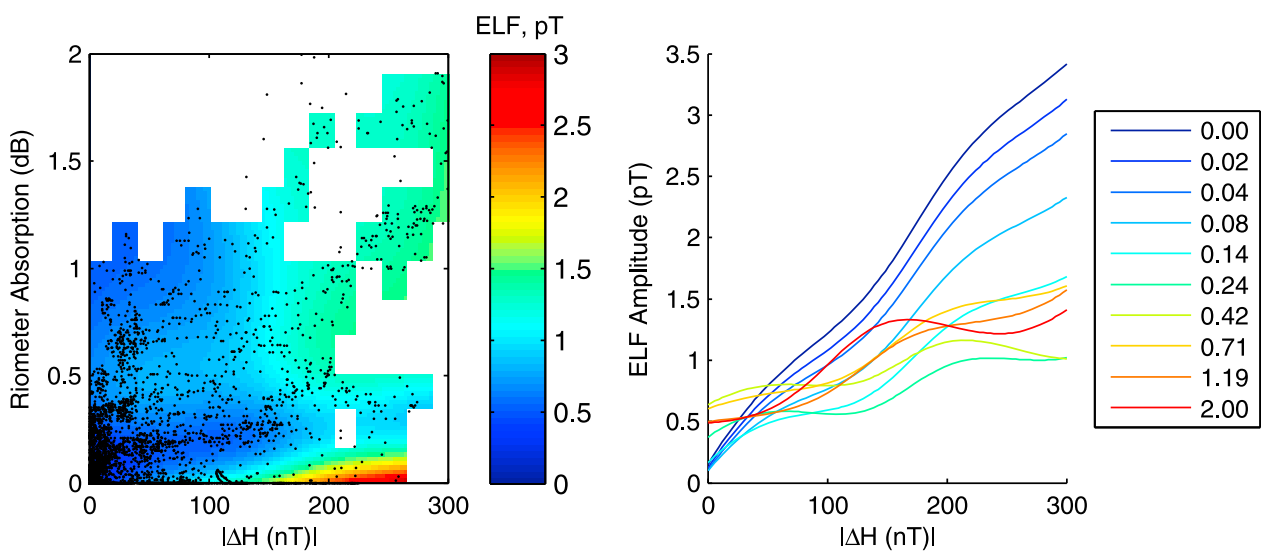

Figure 3. Output of nighttime neural network model showing predicted ELF amplitude versus input values of riometer absorption and $|\Delta H|$. ELF amplitude increases with $|\Delta H|$ but more so with lower absorption values. Black dots indicate the measurements that were used to train the model. ELF versus $|\Delta H|$ shows decreasing slope as riometer absorption increases.

ever, using the equivalent current at HAARP's latitude instead of the Gakona magnetometer resulted in slightly worse correlation between the statistical models and data (e.g., 0.75 versus 0.87 when also using riometer data). The magnetometer has better time resolution, and sits directly under the heated region and is thus more sensitive to local variations in the current instead of being a single current estimate over a swath of latitude. Thus we use $|\Delta H|$ in all the statistical models instead of the computed electrojet current.

[21] We present detailed results of three models, the $|\Delta H|$ and riometer model for both daytime and nighttime and the $|\Delta H|$ and $f_{0} E s$ model for nighttime. Models with more than two input variables are more difficult to visualize and not discussed.

\subsection{Riometer Results}

[22] The results of the nighttime neural network model using magnetometer and riometer data as input show that for a given value of $\Delta H$, lower values of riometer absorption result in stronger ELF amplitudes. Figure 3 shows the output of the model, ELF amplitude, by color plotted against input values of $|\Delta H|$ and absorption. The black dots indicate the measurements that were used to train the model, and the model output is blanked out where there was insufficient input training data. The statistical model shows that the strongest ELF occurs when $\Delta H$ is high and riometer absorption is near zero. Figure 3 also plots the predicted ELF intensity against $\Delta H$ at 10 riometer values. ELF increases roughly linearly with $\Delta H$ with all lines crossing near the origin as expected. As riometer absorption increases from $0 \mathrm{~dB}$ to $\sim 0.2 \mathrm{~dB}$, the slope of the lines decreases from $0.01 \mathrm{pT} / \mathrm{nT}$ to $0.005 \mathrm{pT} / \mathrm{nT}$. As the absorption increases further, the slope stops decreasing and starts increasing again to roughly $0.007 \mathrm{pT} / \mathrm{nT}$ although this is still smaller than the slopes at low absorption values less than $0.1 \mathrm{~dB}$. The model may be less accurate at higher absorption values because less data are available. The range of slopes is consistent with those found by least squares fitting to $18 \mathrm{~min}$ blocks of data shown by Jin et al. [2009].

[23] The results of the daytime model in Figure 4 again with $\Delta H$ and riometer shows similar results. Because the range of $\Delta H$ and absorption values is more limited, we only show the output to $100 \mathrm{nT}$ of $|\Delta H|$ and $1 \mathrm{~dB}$ of absorption.
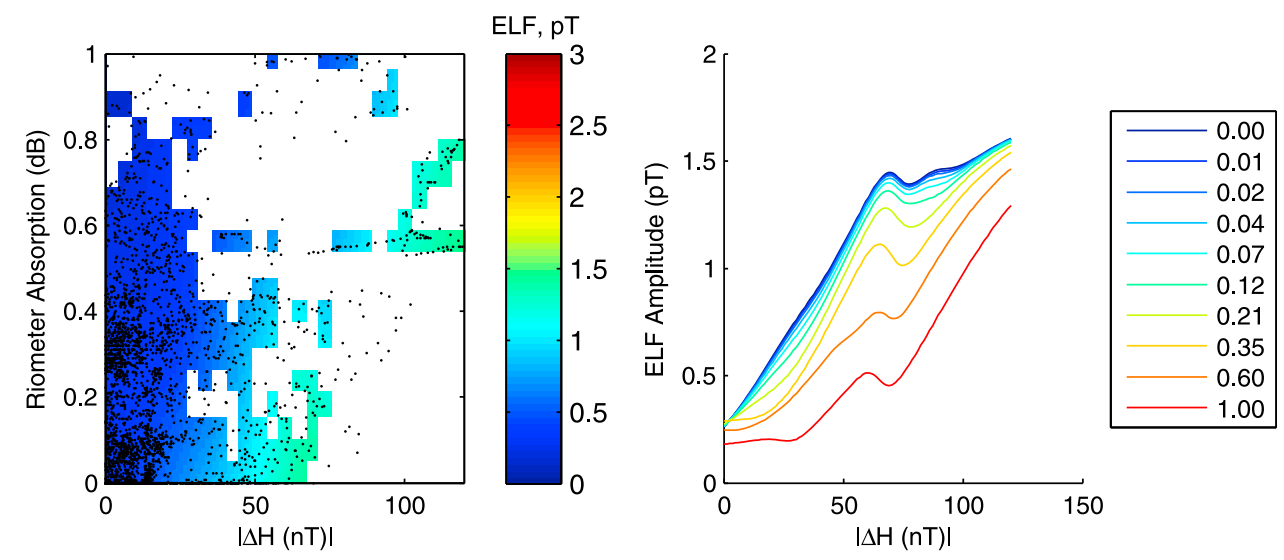

Figure 4. Output of daytime neural network model as in Figure 3. ELF still increases with $|\Delta H|$ with decreasing slope as riometer absorption increases. 

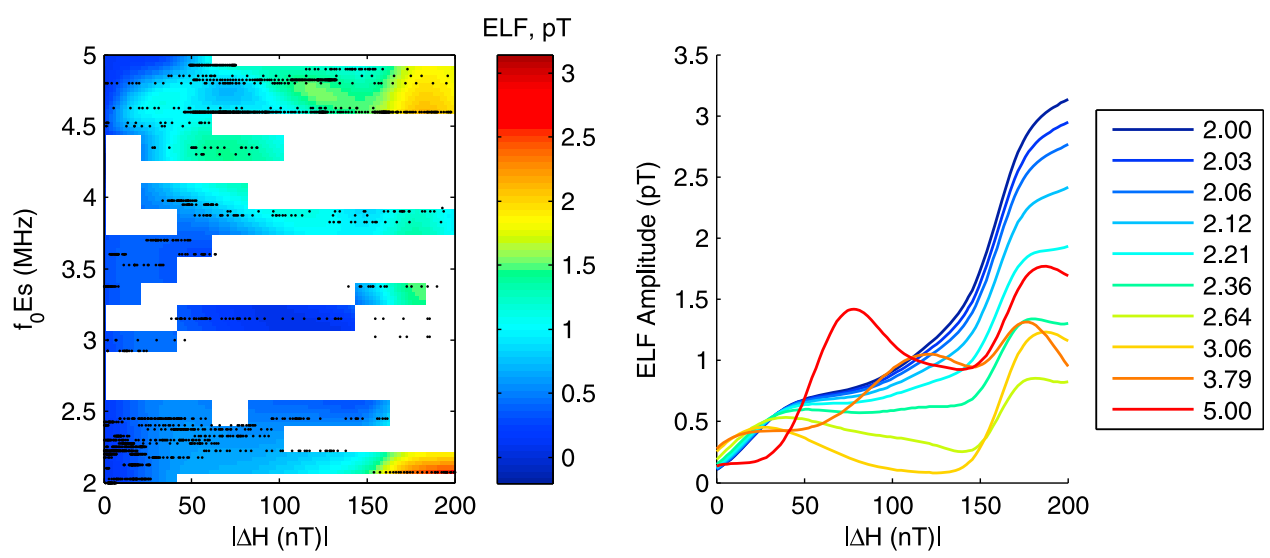

Figure 5. Output of nighttime neural network model showing predicted ELF amplitude versus input values of sporadic $E$ critical frequency and $|\Delta H|$. ELF amplitude increases with $|\Delta H|$ but more so with lower critical frequencies. Black dots indicate the measurements that were used to train the model. ELF versus $|\Delta H|$ shows decreasing slope as critical frequency increases.

ELF amplitude still linearly increases with $|\Delta H|$, with higher absorption values corresponding to smaller proportionality constants.

\subsection{Ionosonde Results}

[24] The result of the nighttime neural network using $\Delta H$ and sporadic $E$ critical frequency $\left(f_{0} E s\right)$ is shown in Figure 5. The results are similar to the neural network model using riometer absorption where the strongest ELF for a given $|\Delta H|$ occurs at smaller values of $f_{0} E s$ and ELF increases with $|\Delta H|$ with a proportionality constant that decreases as $f_{0} E s$ increases. The slopes of the lines decrease from $0.016 \mathrm{pT} / \mathrm{nT}$ to $0.005 \mathrm{pT} / \mathrm{nT}$ as $f_{0} E s$ increases from $1 \mathrm{MHz}$ to $2.6 \mathrm{MHz}$ before increasing slightly to $0.008 \mathrm{pT} / \mathrm{nT}$ as $f_{0} E s$ reaches $5 \mathrm{MHz}$. This is consistent with higher $E$ region densities favoring strong electrojets and $\Delta H$ more than ELF generation in the lower $D$ region resulting in lower proportionality constants. Direct examination of ionograms for a large number of cases could lead to a more nuanced understanding of what ionospheric conditions lead to better ELF generation.

\section{Experimental Summary}

[25] Both the $|\Delta H|$-Riometer and $|\Delta H|$-Ionosonde statistical models show that ELF intensity increases with $|\Delta H|$ for any fixed value of riometer absorption or $f_{0} E s$, respectively. How much ELF increases with $|\Delta H|$ depends on riometer absorption and $f_{0} E s$, where higher values of either lead to smaller increases in ELF intensity (smaller ELF/ $|\Delta H|$ slopes) and smaller values lead to larger increases in ELF (larger ELF/ $\triangle \Delta H \mid$ slopes). Both riometer absorption and $f_{0} E s$ are measurements of density in the lower ionosphere, indicating that for a given electrojet current, one flowing through a less dense ionosphere is more favorable for ELF generation than one flowing through a more dense ionosphere. This is also consistent with Jin et al. [2009], which showed ELF/I $\Delta H \mathrm{l}$ slope decreasing as peak values of $|\Delta H|$ in an interval increased as peak $|\Delta H|$ and density in the lower ionosphere are both associated with increased geomagnetic activity.

\section{Theoretical Modeling}

\subsection{Methodology}

[26] For a theoretical explanation of how ionospheric density affects the electrojet current and ELF amplitude relationship, we model the ELF generation using a HF heating model to determine the ELF source current and a full-wave propagation model [Lehtinen and Inan, 2008] to obtain the ELF source fields on the ground. In addition, we calculate the height integrated steady state electrojet current to determine $\Delta H$, the change in the Earth's north-south magnetic field detected on the ground.

[27] The HF heating component of the model is described in detail by Cohen et al. [2010a] and builds off the work of Tomko et al. [1980], Rodriguez [1994], Payne [2007], and Moore [2007]. To summarize, the three-dimensional model uses a grid, which in this case is defined from $60-95 \mathrm{~km}$ in altitude and spans $160 \mathrm{~km}$ in each horizontal direction. The initial electron density and electron-neutral collision frequency are input at every grid point. A realistic beam pattern of the HAARP array is used to compute the distribution of HF power that enters the bottom layer of the model. The model then computes how much HF power is absorbed. Any remaining power is then propagated to the next higher layer accounting for slowing and refracting the HF beam may have undergone. The modified electron temperature and collision frequency are also calculated at each altitude and utilized in the subsequent time step. The modified kinetic conductivity tensor can also be calculated from the modified collision frequency. When the electron temperature reaches sinusoidal steady state after several ELF periods of heating, the model applies a Fourier series to the modified conductivity in the last ELF period to extract the magnitude and phase of the conductivity at the modulation frequency. Applying Ohm's law to the modified conductivity tensor and an assumed electric field vector yields the modulated currents at every point in the grid.

[28] In order to determine the electromagnetic fields on the ground, the resulting currents are used as an input in a full-wave propagation model [Lehtinen and Inan, 2008]. 

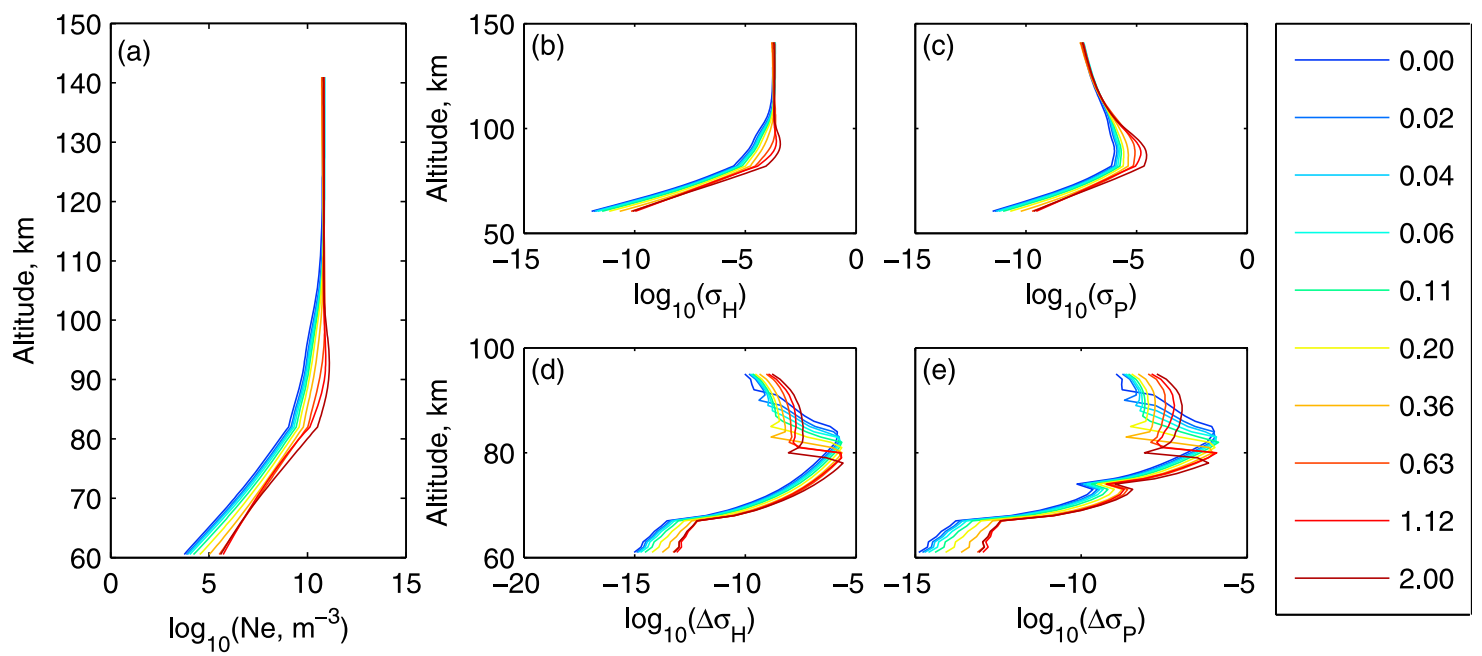

Figure 6. (a) Electron density profiles. (b and c) Resulting Hall and Pedersen ambient conductivities. (d and e) Modulated ELF conductivities from simulation. The ambient conductivities increase with electron density uniformly. Modulated ELF conductivities also increase with electron density with decreasing altitude of peak modulation.

The currents are placed in a larger three-dimensional grid. However, the ambient ionospheric parameters in this model are horizontally stratified and not varying across space or time. This allows for using Snell's Law in a plane-stratified medium to compute reflection coefficients and mode amplitudes for each horizontal wave vector component. The horizontal wave vector components are found from a spatial Fourier transform over the horizontal currents. An inverse Fourier transform of the computed modes then returns the electric and magnetic fields over the three-dimensional grid. The restriction to plane-stratified mediums is mitigated by our intent to simulate fields at Chistochina, only $37 \mathrm{~km}$ from HAARP where the ionosphere is not likely to vary substantially along the propagation path. The restriction also allows the model to be run more quickly than other techniques such as finite difference time domain simulations, important when performing multiple simulations.

[29] Lastly, the ambient electrojet conductivity tensor is calculated directly from the electron density and collision rates of the ionosphere assuming they do not vary across the horizontal direction. Assuming an electric field and applying Ohm's law gives the ambient currents. These are the DC currents of the electrojet and entirely separate from the ELF source current computed using the HF heating model. The ambient currents are integrated in height and scaled according to Kamide et al. [1982] to obtain a simulated value for $\Delta H$ on the ground.

\subsection{Model Inputs}

[30] Since both the ELF source currents and the electrojet current scale linearly with the electric field, we assume a constant (geomagnetic) northward electric field of $1 \mathrm{mV} / \mathrm{m}$ and assume that changes in ELF generation and electrojet current are due to changes in the electron density profile. Density changes will affect the ELF conductivity modulation and ambient conductivity differently and thus the ELF and ambient currents differently. Because the $D$ region is poorly understood, reliable electron density profiles do not exist. Three profiles that have provided good agreement with experimental data in midlatitude lightning studies are Profiles I, II, and III in the work by Pasko and Inan [1994]. The empirical model of McKinnell and Friedrich [2007] is also available and uses solar zenith angle, riometer absorption, $A p$ index, and $F 10.7$. The model incorporates data from incoherent scatter radars, which are plentiful at higher altitudes, but below $60 \mathrm{~km}$, data are sourced from small numbers of rocket measurements. As a compromise, we use the McKinnell and Friedrich [2007] model but replace electron densities below $82 \mathrm{~km}$ with an exponentially decreasing profile consistent with Profiles I, II, and III. As an input to the auroral ionosphere model, we use the latitude and longitude from HAARP and time of 15 March 2008, 01:00 UT and a $F 10.7$ value of 140 . The actual $F 10.7$ values through 2008 were much lower due to an extended solar quiet so a higher value was chosen as the McKinnell and Friedrich [2007] model has better data coverage at the higher $F 10.7$ values. Ten ionospheric profiles were then created by selecting 10 values of riometer absorption logarithmically spaced between 0 and $2 \mathrm{~dB}$ and corresponding values of $A p$ linearly spaced between 0 and 30. Figure $6 a$ shows each of the 10 electron density profiles colored by the riometer absorption value used to create them. Higher values of absorption generally lead to higher electron densities. The HF heating model then determines the electronneutral collision frequency using an empirical formula from Banks [1966] and a neutral density profile from the MSIS model. The HF heating simulation is conducted with a vertical full-power beam profile at $2.75 \mathrm{MHz}$ in $\mathrm{X}$ mode, consistent with the experiments used in the data set.

[31] The same electron density profiles are used in the HF heating model, full-wave propagation model, and the ambient current calculation. In all cases we assume the ionosphere does not vary horizontally at a fixed altitude. We perform the HF simulation at altitudes from $60-95 \mathrm{~km}$ where the majority of the ELF currents are produced. The ambient current calculations are performed at a higher alti- 


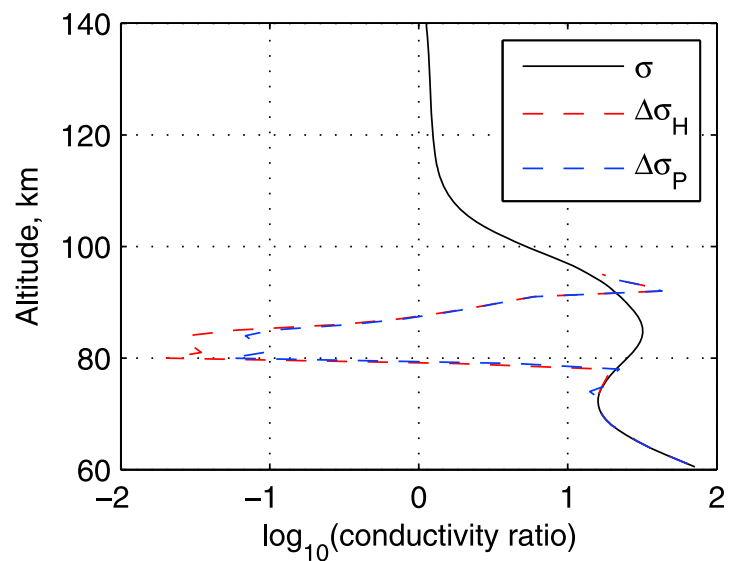

Figure 7. The ratio between simulated conductivities for the most dense ionosphere to conductivities for the least dense ionosphere versus altitude. The ambient Hall and Pedersen conductivities scale with electron density in the same way and are higher with the most dense ionosphere at all altitudes. The modulated Hall and Pedersen conductivities do not improve as much as the ambient conductivities.

tude range from $60-140 \mathrm{~km}$ (the maximum valid altitude of the ionospheric density model) to include the higher altitude range the electrojet flows through.

\subsection{Model Results}

[32] Figures $6 \mathrm{~b}$ and $6 \mathrm{c}$ show the Hall and Pedersen conductivities, respectively, computed directly from each of the electron density profiles in Figure 6a. Both conductivities are higher when the electron densities also increase, with the Hall conductivity being more dominant than the Pedersen conductivity especially above $100 \mathrm{~km}$. Thus, elevated ionospheric densities indicated by higher riometer absorption leads directly to elevated ambient conductivities in the ionosphere and stronger currents for a given electric field.

[33] Figures 6d and 6e show the modified Hall and Pedersen conductivities from the HF heating model at each altitude in the center of the HAARP beam. In contrast to the ambient conductivities these do not show an obvious trend as ionospheric density increases. The modified Hall conductivity is generally larger than the Pedersen conductivity and increases with altitude before reaching a maximum near 80 $90 \mathrm{~km}$ after which it drops off as most of the HF power has already been absorbed. The altitude of this maximum decreases for profiles with higher ionospheric density a result consistent with Payne [2007] and Barr and Stubbe [1984]. Current sources lower in altitude typically improve coupling into the Earth-ionosphere waveguide. However, while the magnitude of the modified Hall conductivity increases with higher ionospheric density below the peak altitude, above the peak the modified conductivity decreases more rapidly for the denser profiles. This is more apparent in Figure 7, which shows the ratio between the conductivities simulated with the most dense profile and least dense profile. For example a ratio of 1 indicates that the conductivity for the most dense profile is the same as for the least dense profile. The ambient conductivity ratio is shown in the solid line and is the same for both the Hall and Pedersen conductivities as they both respond to electron density in the same way. The ambient conductivity is an order of magnitude larger for the densest profile compared to the least dense profile below $95 \mathrm{~km}$. The ratio for the modulated conductivity is smaller than the ambient conductivity. The densest ionosphere actually produces less conductivity modulation than the least dense ionosphere above $80 \mathrm{~km}$ (ratio less than 1). At most altitudes, the ambient conductivity benefits more from a denser ionosphere than the modulated conductivity. To directly compare the effect of different density profiles on the ambient DC electrojet current and the modulated ELF source current, we use the results of the full-wave model to obtain the magnetic fields on the ground.

[34] The outputs of the full-wave model are shown in Figure 8a along with the simulated value of $|\Delta H|$ obtained from the ambient conductivities. Each point corresponds to one of the ionospheric density profiles from Figure $6 \mathrm{a}$ with its position showing the resulting ELF amplitude from the full-wave model against the resulting $|\Delta H|$ from the ambient currents when normalized using an electric field of $1 \mathrm{mV} / \mathrm{m}$. The points corresponding to the denser profiles show increased ELF amplitudes, but this increase is smaller than increases in $|\Delta H|$. For example, the point furthest right has a $|\Delta H|$ value over twice as large as the point furthest to the left but an ELF amplitude only 1.6 times as large.

[35] Since changes in the electric field would affect both the ELF and electrojet fields linearly, the proportionality constant between ELF and $|\Delta H|$ is fixed for a given density and found by dividing the normalized ELF fields by the normalized electrojet field. Figure $8 \mathrm{~b}$ shows the ELF/l $\Delta H \mathrm{l}$ slope, ranging from $0.024 \mathrm{pT} / \mathrm{nT}$ for an absorption value of
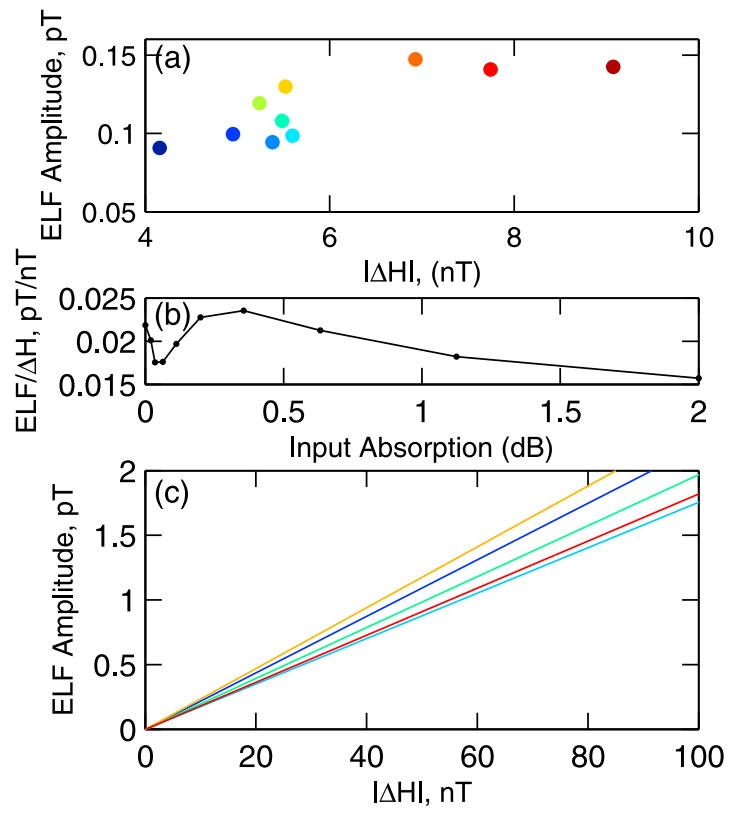

Figure 8. (a) Simulated ELF amplitude versus $|\Delta H|$ using $E=1 \mathrm{mV} / \mathrm{m}$ using ionospheric density profiles generated from riometer absorption values. (b) ELF amplitude/l $\Delta H \mathrm{l}$ slope versus the riometer absorption values. $|\Delta H|$ increases more quickly with higher absorption than ELF amplitude, resulting in decreasing slope of ELF/I $\Delta H \mathrm{I}$. (c) How ELF amplitude and $|\Delta H|$ vary for each ionosphere as electric field changes. For clarity only half the lines are shown. 
$0.36 \mathrm{~dB}$ to $0.016 \mathrm{pT} / \mathrm{nT}$ for an absorption value of $2 \mathrm{~dB}$. Figure 8c shows an ELF versus $|\Delta H|$ plot for half of the different ionospheric profiles. This plot is similar to Figure $8 \mathrm{a}$ except that each point now sweeps out a line when the electric field is varied. The slope of each line generally decreases with denser ionospheric density.

[36] This range of proportionality constants is consistent with that determined statistically. This also accounts for the two distinct regimes in the example of 15 March 2008 from Figure 1, where the quiet period corresponds to a higher proportionality constant and a less dense ionosphere, and the disturbed period to a lower proportionality constant and a more dense ionosphere. There is however an increase in slope with higher riometer absorption when the absorption was between 0.04 and $0.36 \mathrm{~dB}$. This is likely due to the ionospheric density profiles used. The density at low altitudes increases with higher absorption, but it can decrease at higher altitudes (Figure 6a) with the result that the height-integrated conductivity fails to increase as much as expected. ELF generation improves slightly from the enhanced low-altitude density while $\mid \Delta H \mathrm{I}$ remains roughly constant leading to the increasing slope in this regime. There is still an overall trend of decreasing slope with higher absorption.

\section{Discussion}

[37] The results from the statistical and theoretical models can provide insight into how natural ionospheric conditions affect ELF generation. At nighttime, in the simplest case, if ionospheric density is steady and there are only variations in the electric field, then ELF generation and magnetometer measurements will be well correlated. The proportionality constant between the two is then related to ionospheric density, which can be estimated using riometer absorption. The statistical model showed this as a linear increase in ELF intensity with $|\Delta H|$ for a fixed value of riometer absorption.

[38] Rapidly changing densities in the ionosphere however can result in much less predictable behavior especially if the electric field is also changing. In the statistical model, this manifests as a decrease in the ELF/l $\Delta H \mathrm{l}$ proportionality constant during periods of high riometer absorption or high sporadic $E$ critical frequency. During these periods of high absorption, changes in ELF intensity are smaller than changes in $|\Delta H|$. The theoretical model shows the same result and confirmed that the effect was due to a denser ionosphere resulting in increases in ambient conductivity and $|\Delta H|$ but smaller increases or decreases in HF modulated conductivities and ELF. Both the statistical and theoretical models predict similar changes in the ELF/I $\Delta H \mathrm{l}$ proportionality constant from a range of $0.01-0.005 \mathrm{pT} / \mathrm{nT}$ for the statistical model to a range of $0.024-0.016 \mathrm{pT} / \mathrm{nT}$ for the theoretical model.

[39] The discrepancy between the values of the proportionality constant from the theoretical and statistical models is likely due to underestimation of the magnetometer measurement in the theoretical model. The ionospheric profiles from McKinnell and Friedrich [2007] stop near $140 \mathrm{~km}$ though currents above that altitude may still contribute to magnetic deviations on the ground. It is also possible that the statistical model is underestimating the proportionality constant at very low absorption values. In quiet cases where background conductivity is very low, but electric fields are changing substantially, ELF amplitude can change significantly despite little response in the electrojet and thus magnetometer. In such cases, it does not make sense to assign a proportionality constant between ELF amplitude and $|\Delta H|$ as the two quantities are essentially uncorrelated and a neural network model will tend to simply give an average value for the ELF output. If such a proportionality constant were to be assigned, it would approach infinity and a plot of ELF amplitude versus $\mid \Delta H \mathrm{I}$ (e.g., Figure 8c) would be a vertical line. These conditions are conducive to strong ELF generation despite little signature of an electrojet on magnetometers because of very low conductivity. Examples of such conditions can be found in Kirkwood et al. [1988], which also concludes that short intervals of strong electric fields can precede substorm onset by 2-20 min, a result consistent with the sudden increase in ELF intensity before substorm onset in Figure 1.

[40] Results from the theoretical model may also explain negative correlation between magnetometer measurements and ELF generation such as reported by Jin et al. [2009]. For example if the electron density (and ambient conductivity) increase substantially, but the electric field weakens slightly, the electrojet may intensify as the increased conductivity overcomes the reduced electric field while ELF generation weakens as the decreased electric field overcomes any gain in modulated conductivities. There are points in Figure $8 \mathrm{a}$, including the second and third points, where for a fixed electric field, ELF intensity actually decreases when $\mid \Delta H \mathrm{l}$ increases, allowing for negative correlation if the ionosphere is changing in this region without any change in the electric field.

[41] Radar measurements of electric field over HAARP would help complete our understanding of how ELF generation is affected by natural conditions. However, it may also be possible for ELF amplitude to be used in an inverse model to determine electric fields when the ionosphere is not significantly changing. ELF generation is reliable and sensitive to electric fields even when magnetometers do not detect any electrojet and riometers do not detect any absorption making it a potentially powerful diagnostic.

\section{Conclusion}

[42] We have presented both statistical and theoretical models showing that ELF amplitude is linearly related to $|\Delta H|$. At nighttime the proportionality constant can be determined by riometer absorption or sporadic $E$ layer critical frequency and decreases as absorption or critical frequency increases. During disturbed conditions, the $E$ region electron density and conductivity increases resulting in a much stronger electrojet and higher $|\Delta H|$. Even if $D$ region density also increases, the gain in ELF intensity is much smaller. Increases in electric field which also contribute to the stronger electrojet are the larger factor in better ELF generation during increased geomagnetic activity. During the daytime, $E$ layer critical frequency is a slightly better diagnostic of ELF generation than riometer absorption.

[43] Acknowledgments. This research has been carried out with support from HAARP, Office of Naval Research, Air Force Research Laboratory, and Defense Advanced Projects Research Agency, via 
ONR grants N0001405C0308 and N00014091 to Stanford University, and by a Stanford Graduate Fellowship. Magnetometer data are provided by the Geophysical Institute, University of Alaska, Fairbanks. We also thank Mike McCarrick and Helio Zwi for operating the HAARP array as well as Jens Østergaard for maintaining the HAARP riometer and Bill Bristow for assisting with SuperDARN data.

[44] Robert Lysak thanks the reviewers for their assistance in evaluating this paper.

\section{References}

Banks, P. (1966), Collision frequencies and energy transfer electrons, Planet. Space Sci., 14, 1085-1103, doi:10.1016/0032-0633(66)90024-9.

Barr, R., and P. Stubbe (1984), ELF and VLF radiation from the "polar electrojet antenna," Radio Sci., 19, 1111-1122.

Chisham, G., et al. (2007), A decade of the Super Dual Auroral Radar Network (SuperDARN): Scientific achievements, new techniques and future directions, Surv. Geophys., 28, 33-109, doi:10.1007/s10712-007-9017-8.

Cohen, M. B., U. S. Inan, M. Gołkowski, and N. G. Lehtinen (2010a), On the generation of ELF/VLF waves for long-distance propagation via steerable HF heating of the lower ionosphere, J. Geophys. Res., 115, A07322, doi:10.1029/2009JA015170.

Cohen, M. B., U. S. Inan, M. Gołkowski, and M. J. McCarrick (2010b), ELF/ VLF wave generation via ionospheric HF heating: Experimental comparison of amplitude modulation, beam painting, and geometric modulation, J. Geophys. Res., 115, A02302, doi:10.1029/2009JA014410.

Cohen, M. B., U. S. Inan, and E. W. Paschal (2010c), Sensitive broadband ELF/VLF radio reception with the AWESOME instrument, IEEE Trans. Geosci. Remote Sens., 48, 3-17, doi:10.1109/TGRS.2009.2028334.

Fine, T. L. (1999), Feedforward Neural Network Methodology, Statistics for Engineering and Information Science, edited by J. Fan and Q. Yao, Springer, New York, doi:10.1007/b97705.

Getmantsev, G. G., N. A. Zuikov, D. S. Kotik, L. F. Mironenko, N. A Mitiakov, V. O. Rapoport, Y. A. Sazonov, V. Y. Trakhtengerts, and V. Y. Eidman (1974), Combination frequencies in the interaction between high-power short-wave radiation and ionospheric plasma, Sov. Phys. JETP, Engl. Transl., 20, 229-232.

Hagan, M. T., and M. B. Menhaj (1994), Training feedforward networks with the Marquardt algorithm, IEEE Trans. Neural Networks, 5(6), 989-993.

Jin, G., M. Spasojevic, and U. S. Inan (2009), Relationship between electrojet current strength and ELF signal intensity in modulated heating experiments, J. Geophys. Res., 114, A08301, doi:10.1029/ 2009JA014122.

Kamide, Y., S.-I. Akasofu, B.-H. Ahn, W. Baumjohann, and J. L. Kisabeth (1982), Total current of the auroral electrojet estimated from the IMS Alaska meridian chain of magnetic observatories, Planet. Space Sci., 30, 621-625, doi:10.1016/0032-0633(82)90022-8.

Kapustin, I. N., R. A. Pertsovskii, A. N. Vasil'Ev, V. S. Smirnov, O. M. Raspopov, L. E. Solov'eva, A. A. Ul'Yachenko, A. A. Arykov, and N. V. Galakhova (1977), Generation of radiation at combination fre- quencies in the region of the auroral electric jet, Sov. Phys. JETP, Engl. Transl., 25, 228-231.

Kirkwood, S., H. Opgenoorth, and J. S. Murphree (1988), Ionospheric conductivities, electric fields and currents associated with auroral substorms measured by the EISCAT radar, Planet. Space Sci., 36, 1359-1380, doi:10.1016/0032-0633(88)90005-0.

Lehtinen, N. G., and U. S. Inan (2008), Radiation of ELF/VLF waves by harmonically varying currents into a stratified ionosphere with application to radiation by a modulated electrojet, J. Geophys. Res., 113, A06301, doi:10.1029/2007JA012911.

McKinnell, L.-A., and M. Friedrich (2007), A neural network-based ionospheric model for the auroral zone, J. Atmos. Sol. Terr. Phys., 69 , 1459-1470, doi:10.1016/j.jastp.2007.05.003.

Moore, R. C. (2007), ELF/VLF wave generation by modulated HF heating of the auroral electrojet, Ph.D. thesis, Stanford Univ., Stanford, Calif.

Oikarinen, A., J. Manninen, J. Kultima, and T. Turunen (1997), Observations of intensity variations and harmonics of heater induced VLF waves, J. Atmos. Sol. Terr. Phys., 59, 2351-2360.

Pasko, V. P., and U. S. Inan (1994), Recovery signatures of lightningassociated VLF perturbations as a measure of the lower ionosphere, J. Geophys. Res., 99, 17,523-17,538.

Payne, J. A. (2007), Spatial structure of very low frequency modulated ionospheric currents, Ph.D. thesis, Stanford Univ., Stanford, Calif.

Rietveld, M. T., H. Kopka, E. Nielsen, P. Stubbe, and R. L. Dowden (1983), Ionospheric electric field pulsations: A comparison between VLF results from an ionospheric heating experiment and STARE, $J$. Geophys. Res., 88, 2140-2146.

Rietveld, M. T., H.-P. Mauelshagen, P. Stubbe, H. Kopka, and E. Nielsen (1987), The characteristics of ionospheric heating-produced ELF/VLF waves over 32 hours, J. Geophys. Res., 92, 8707-8722.

Rodriguez, J. V. (1994), Modification of the Earth's ionosphere by verylow-frequency transmitters, Ph.D. thesis, Stanford Univ., Stanford, Calif.

Ruohoniemi, J. M., and K. B. Baker (1998), Large-scale imaging of highlatitude convection with Super Dual Auroral Radar Network HF radar observations, J. Geophys. Res., 103, 20,797-20,811.

Shepherd, S. G., and J. M. Ruohoniemi (2000), Electrostatic potential patterns in the high-latitude ionosphere constrained by SuperDARN measurements, J. Geophys. Res., 105, 23,005-23,014.

Sun, W., Y. Kamide, J. R. Kan, and S.-I. Akasofu (1993), Inversion of the auroral electrojets from magnetometer chain data based on the Flexible Tolerance method, J. Geomagn. Geoelectr., 45, 1151.

Tomko, A. A., A. J. Ferraro, and H. S. Lee (1980), D region absorption effects during high-power radio wave heating, Radio Sci., 15, 675-682.

Wilkinson, D., and M. J. Heavner (2006), Geophysical Institute Magnetometer Array, EOS Trans. AGU, 87(52), Fall Meet. Suppl., Abstract SA41B-1417.

M. B. Cohen, U. S. Inan, G. Jin, N. G. Lehtinen, and M. Spasojevic, Department of Electrical Engineering, Stanford University, 350 Serra Mall, Rm. 356, Stanford, CA 94305, USA. (gj36@stanford.edu) 